\title{
Recurrencia de miocardiopatía por estrés posterior a terremoto en Chile. Reporte de un caso clínico
}

\author{
CECILIA VARELA U. ${ }^{1, a}$, RICARDO BOHN R., ${ }^{1, a}$, \\ PAOLA VARLETA O. ${ }^{2}$, ROBERTO CONCEPCIÓN C. ${ }^{2}$
}

\section{Recurrence of stress cardiomyopathy after an earthquake. Report of one case}

\begin{abstract}
Stress-induced cardiomyopathy is characterized by transient systolic dysfunction of the apical or mid segments of the left ventricle that mimics myocardial infarction in the absence of obstructive coronary artery disease. Symptoms recur after a physical or emotional stress. We report a 77 years old female that in 2004, suffered an episode of stress cardiomyopathy after an intense physical effort. In February 2010, immediately after the earthquake that occurred in Chile, the patient consulted for chest pain, ST segment elevation and enzyme elevation. An echocardiography showed a left ventricular anteroseptal akinesia with an ejection fraction of 35\%. Coronary arteriography did not show significant alterations.
\end{abstract}

(Rev Med Chile 2011; 139: 79-83).

Key words: Cardiomyopathies; Stress, psychological; Ventricular dysfunction, left.

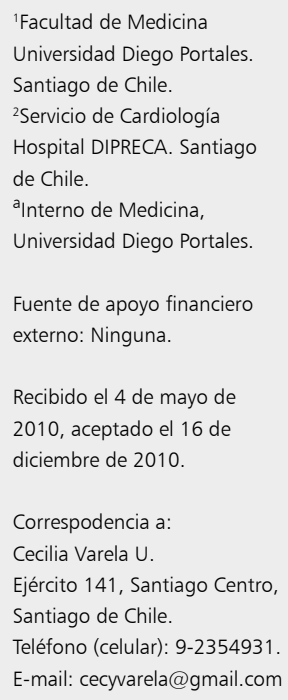

I a miocardiopatía por estrés es una enfermedad descrita en 1990 en población japonesa. Consiste en una disfunción sistólica apical del ventrículo izquierdo reversible, precipitada por situaciones de estrés emocional o físico, con una forma de presentación clínica indistinguible del infarto agudo de miocardio (IAM).

Se presenta el caso de una paciente mujer con dos episodios de miocardiopatía por estrés, el último evento después del terremoto de la madrugada del 27 de febrero de 2010.

\section{Caso clínico}

Mujer de 77 años con antecedentes de hipertensión arterial en tratamiento con carvedilol y dislipidemia, que consultó por primera vez en abril de 2004 por un cuadro de dolor opresivo retroesternal mientras caminaba aproximadamente 20 cuadras, esfuerzo inusual para ella. Se hospitalizó en la Unidad Coronaria 6 horas después de haber iniciado los síntomas. En los exámenes de ingreso presentó: Electrocardiograma (ECG) con inversión simétrica de la onda T en V1-V6, troponina $\mathrm{T} 0,578 \mathrm{ng} / \mathrm{mL}(0,00-0,03), \mathrm{CK}$ total $163 \mathrm{ng} / \mathrm{mL}$ (30-115), CK-MB 22 ng/mL (0-16). Se trató como IAM sin elevación del ST. Al día siguiente se realizó ecocardiograma que reveló disfunción sistólica severa del ventrículo izquierdo y aquinesia extensa anteroseptoapical, con fracción de eyección de $35 \%$. Se realizó coronariografía que no mostró lesiones angiográficas significativas, diagnosticándose disfunción sistólica transitoria del ventrículo izquierdo (miocardiopatía por estrés). La paciente evolucionó satisfactoriamente, con regresión de onda $\mathrm{T}$, prolongación transitoria del intervalo QTc y descenso de marcadores séricos cardiacos. Egresó estable, asintomática.

En el año 2006, en forma ambulatoria, se realizó un ecocardiograma de control que reveló motilidad global y segmentaria del ventrículo izquierdo conservada y fracción de eyección sobre $60 \%$.

El 27 de febrero del año 2010, estando en la ciudad de Santiago, la paciente comenzó con un 
cuadro de angina de reposo intensa, con marcados síntomas neurovegetativos a las 3:40 AM, inmediatamente posterior al terremoto. Consultó al Servicio de Urgencia a las 5:45 AM. Al ingreso la paciente se encontraba hipertensa y taquicárdica. El ECG mostró supradesnivel del segmento ST en V1-V3 (Figura 1a), troponina T 0,184 ng/mL, CK total $118 \mathrm{ng} / \mathrm{mL}$ y CK-MB 18,4 ng/mL. Se decidió realizar trombolisis con estreptoquinasa 1.500 .000 UI intravenosa en 45 minutos, procedimiento sin incidentes. La paciente evolucionó sin angor, en clase Killip I, con disminución progresiva de las enzimas cardiacas. Cuarenta y ocho horas posterior al evento inicial, se realizó ecocardiograma que reveló ventrículo izquierdo levemente dilatado en sístole con reducción moderada de motilidad global por aquinesia septoapical y lateral, con fracción de eyección estimada en 35\% (Figura 2a). Posteriormente, se realizó coronariografía que no reveló lesiones angiográficas significativas y la ventriculografía mostró un balonamiento de los segmentos apicales del ventrículo izquierdo con una fracción de eyección estimada de $40 \%$ (Figura 3). El electrocardiograma previo al alta no mostró signos de isquemia (Figura 1b).

La paciente evolucionó en forma satisfactoria, sin angor ni disnea y se decidió el alta a los 5 días post ingreso, quedando con carvedilol, aspirina, plavix, atorvastatina y enalapril en forma permanente.

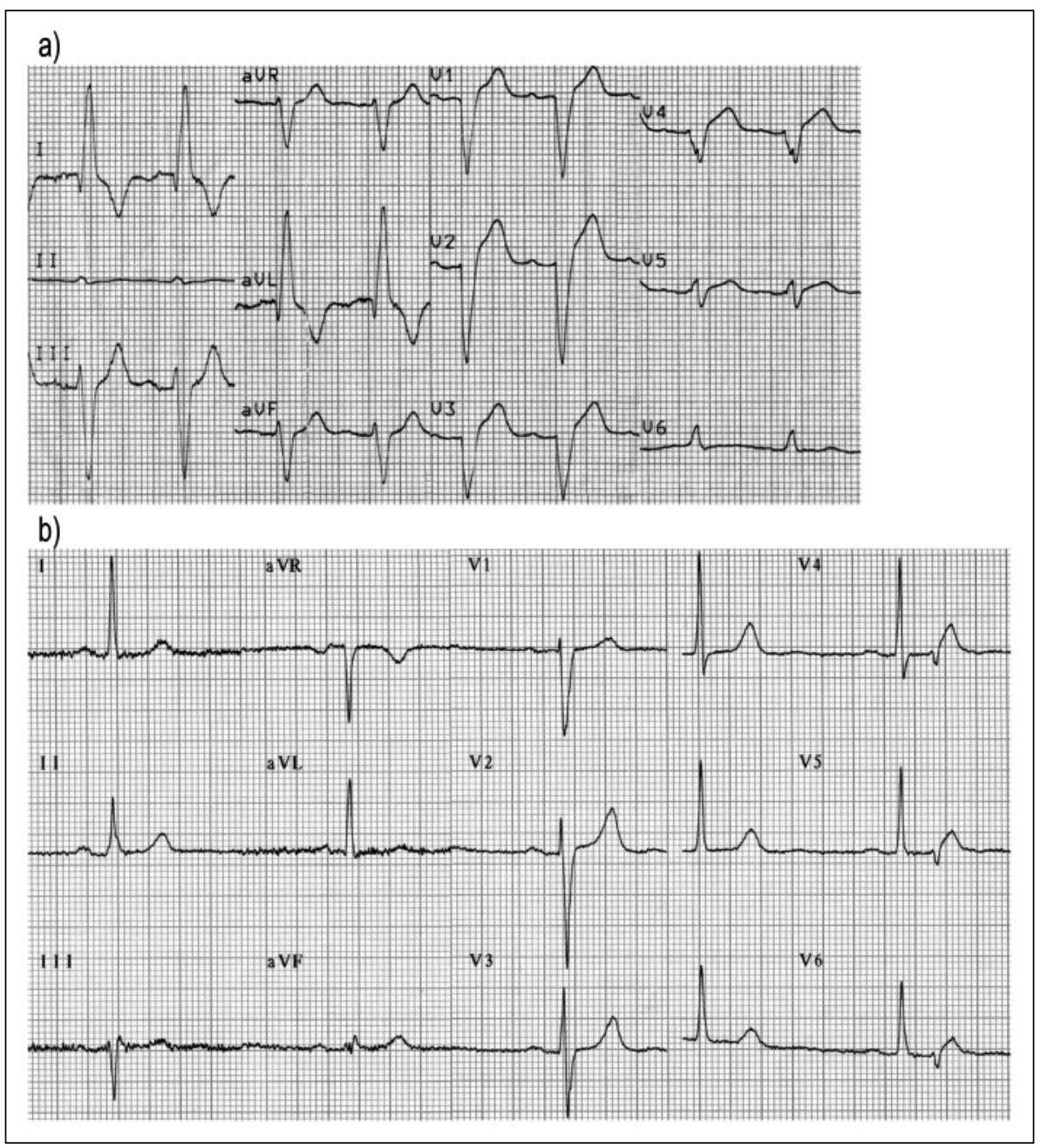

Figura 1. (a) Electrocardiograma de ingreso, con elevación del segmento ST en V1-V3 y alteraciones de la repolarización. (b) Electrocardiograma previo al alta normal. 

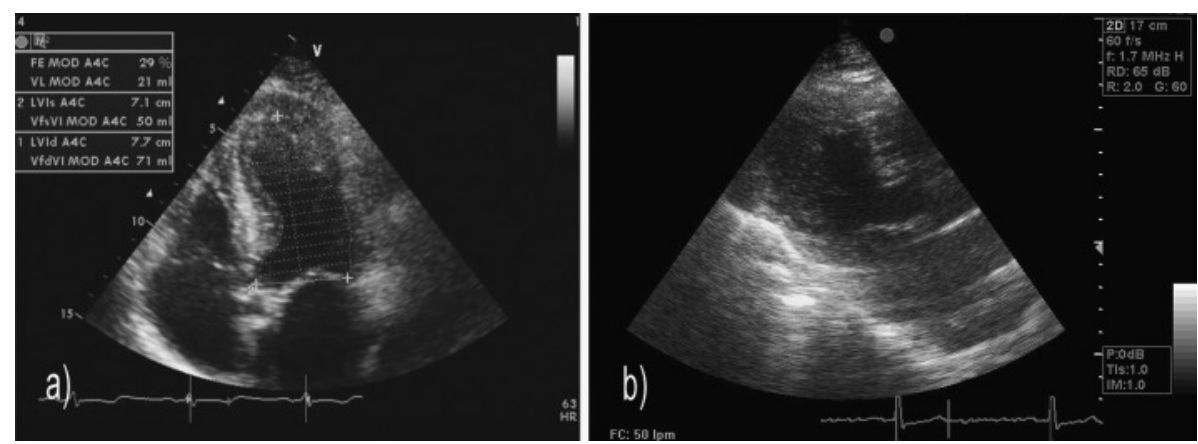

Figura 2. (a) Ecocardiograma 48 horas posterior al evento agudo (visión 4 cámaras) muestra extensa aquinesia septoapical del ventrículo izquierdo. (b) Ecocardiograma de control que muestra contractilidad normal del ventrículo izquierdo.
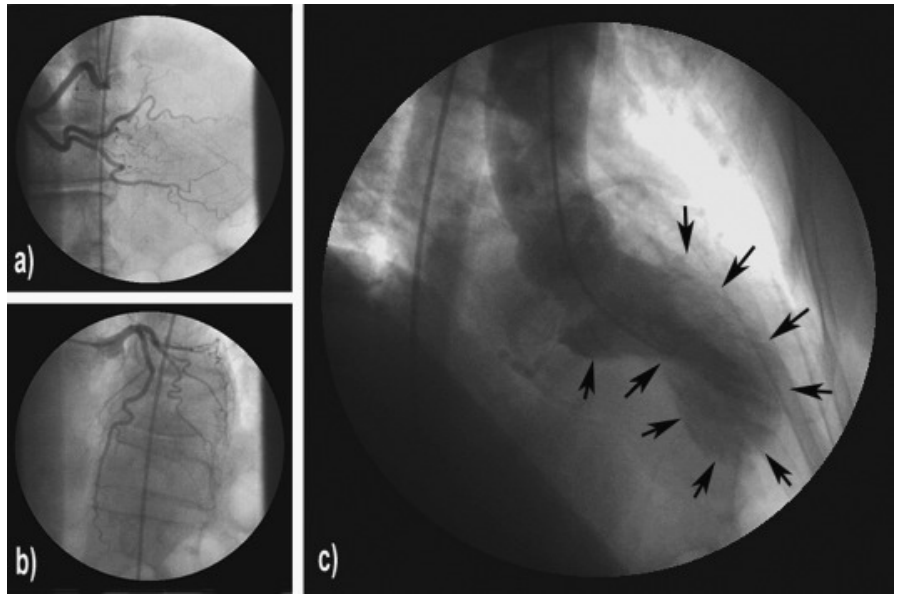

Figura 3. Coronariografía sin lesiones significativas de arterias coronaria derecha (a) e izquierda (b). Ventriculografía (c) destaca balonamiento apical en sístole, flechas indican contorno de cavidad ventricular.

El 18 de junio de 2010, se le realizó un ecocardiograma de control que mostró una fracción de eyección estimada en $66 \%$ y la aurícula izquierda dilatada (Figura 2b).

\section{Discusión}

La miocardiopatía por estrés (ME), también llamada síndrome de balonamiento apical, miocardiopatía Tako-Tsubo o miocardiopatía de la ámpula, fue descrita por primera vez en Japón en 1990. Desde entonces es llamada "miocardiopatía tako-tsubo", nombre del jarrón japonés usado para atrapar pulpos, que tiene el cuello estrecho y el fondo redondo, asemejándose a la ventriculografía en sístole de estos pacientes ${ }^{1}$. En esta patología se revelan anormalidades de la motilidad "tipo globo" en el ápex del ventrículo izquierdo, aunque se describen otras variantes de disfunción ventricular izquierda, como el balonamiento medioventricular que no afecta a los segmentos apicales ni basales, o el tako-tsubo inverso ${ }^{2,3}$.

Los síntomas de la ME ocurren después de un estrés físico o emocional en dos tercios de los pacientes ${ }^{4}$ y son similares a los del IAM, con dolor retroesternal y/o disnea, ambos de inicio repentino y en reposo. El ECG en la fase aguda se presenta con elevación convexa del segmento ST, más comúnmente en V1 a V5, acompañándose de ondas $\mathrm{Q}$ en algunos casos. La evolución electrocardiográfica incluye inversión de ondas $\mathrm{T}$ progresiva (más prominentes en V2 a V6), prolongación del intervalo QTc y reaparición de ondas R. En los exámenes de laboratorio hay un incremento moderado de la creatin kinasa y troponina ${ }^{5}$, siendo entonces indistinguible del IAM. En el caso clínico presentado, la decisión médica de iniciar terapia trombolítica fue justificable ya 
que el clínico carecía del antecedente de ME y estaba frente a una paciente con angina clásica y ECG con supradesnivel del ST. No obstante es necesario destacar que la terapia trombolítica no está indicada en estos pacientes.

La prevalencia de la ME es incierta. Sin embargo, varios estudios han estimado que aproximadamente 1 a $2 \%$ de todos los pacientes con diagnóstico inicial de IAM tienen esta condición. Aproximadamente el $90 \%$ de todos los casos reportados han sido mujeres, la edad media de presentación es entre 58 a 75 años, mujeres postmenopáusicas ${ }^{4}$.

Los mecanismos fisiopatológicos de la ME todavía no son del todo claros. La teoría más aceptada sugiere que la sobrecarga de catecolaminas, precipitada por las situaciones de estrés, ocasionaría las alteraciones de la motilidad observadas, produciendo cambios morfológicos de los cardiomiocitos y alteraciones de la homeostasis del calcio, con disminución de la contractilidad, pero sin cambios apoptóticos ${ }^{6}$. También se ha demostrado que en estos pacientes existe disfunción microvascular miocárdica difusa que podría ser causada por un aumento de la secreción de catecolaminas, induciendo daño microvascular mediante la activación del receptor $1^{6}$.

Los siguientes son los criterios diagnósticos de la Clínica Mayo para ME. Se necesitan los cuatro para el diagnóstico ${ }^{4}$ :

1. Hipokinesia, akinesia o diskinesia transitoria de los segmentos medios del ventrículo izquierdo con o sin involucramiento de la región apical; las anormalidades de la motilidad segmentaria se extienden más allá de la distribución vascular de una sola arteria coronaria; un gatillante de estrés está a menudo presente, pero no siempre. (Hay excepciones donde la anormalidad regional de motilidad puede estar limitada a una sola arteria coronaria).

2. Ausencia de enfermedad coronaria obstructiva o de evidencia de ruptura de una placa. (Puede haber pacientes con ateroesclerosis que desarrollan ME, sin embargo, es muy raro, tal vez porque estos casos son mal diagnosticados como IAM).

3. Anormalidades electrocardiográficas nuevas (elevación del segmento ST y/o inversión de las ondas T) o una elevación modesta de la troponina sérica.

4. Ausencia de feocromocitoma o miocarditis.
La resonancia nuclear magnética (RNM) cardiaca con medio de contraste tendría un rol diferenciador de ME, especialmente con el IAM. Eitel y col, objetivaron la utilidad de la RNM cardiaca en pacientes en que se sospechó ME por criterios de la Clínica Mayo, detectando 22\% de casos compatibles con IAM y $14 \%$ con miocarditis aguda ${ }^{10}$.

El pronóstico de los pacientes con ME es favorable, y de ahí la importancia de considerarlo en la práctica clínica y diferenciarlo del IAM. De 286 pacientes incluidos en una revisión sistemática sólo 3 murieron (dos por falla orgánica múltiple y uno por cáncer de ovario). La insuficiencia cardiaca con o sin edema pulmonar fue la complicación clínica más común, reportada en $17,7 \%$ de los pacientes ${ }^{7}$. La sobrevida en pacientes con ME a 4 años es similar a la esperada para una población pareada por género, edad y etnia ${ }^{8}$ debido a que la alteración de la motilidad es reversible, observándose la recuperación completa de la función sistólica típicamente entre 4 y 6 semanas ${ }^{9}$. No existe consenso sobre el tratamiento crónico de esta condición. La terapia al alta de esta paciente tiene un fundamento en estabilización de la placa ateroesclerótica, disminución de consumo de oxígeno y de trombosis intraarterial. A pesar de que no tenía lesiones significativas coronarias de ateroesclerosis, las causas de esta enfermedad no permiten descartar un componente de angina microvascular en la cual se utiliza la terapia ya mencionada. El $\beta$-bloqueo es además un tratamiento empírico que se ha propuesto con el fin de reducir la probabilidad de una recurrencia al disminuir la respuesta adrenérgica al estrés 4 .

La aparición de un nuevo evento anginoso con las características de $\mathrm{ME}$, en una paciente que ya ha presentado un primer episodio, es inusual. En la literatura no existe documentación que detalle esta situación a excepción del estudio de Elesber y col. que hace referencia a la recurrencia de $\mathrm{ME}$ en un grupo de 100 pacientes con un seguimiento de 4 años, donde fue estimada en $10 \%{ }^{8}$. Nuestra paciente tuvo recurrencia después de 6 años de su primer episodio, algo inédito en la literatura, por esto proponemos que las recurrencias son una característica común de esta patología en el largo plazo. La variabilidad en las recurrencias podría estar dada por la cantidad de factores estresantes durante el resto de la vida, que sean de tal magnitud que sobrepasen un cierto umbral de respuesta adrenérgica para desencadenar la ME. 


\section{Referencias}

1. Sato H, Tateishi H, Uchida T, Dote K, Ishihara M. Takotsubo-like left ventricular dysfunction due to multivessel coronary spasm. En: Kodama K, Haze K, Hori M, editors. Clinical aspect of myocardial injury: from ischemia to heart failure (En Japonés). Tokyo: Kagakuhyoronsha Publishing Co; 1990: 56-64.

2. Botto F, Trivi M, Padilla LT. Transient left midventricular ballooning without apical involvement. Int J Cardiol 2008; 127: 158-9.

3. Marti V, Carreras F, Pujadas S, De Rozas JM. Transient left ventricular basal ballooning-“inverted” Tako-Tsubo. Clin Cardiol 2008; 32: 20-1.

4. Prasad A, Lerman A, Rihal CS. Apical ballooning syndrome (Tako-Tsubo or stress cardiomyopathy): a mimic of acute myocardial infarction. Am Heart J 2008; 155: 408-17.

5. Sharkey SW, Lesser JR, Menon M, Parpart M, Maron MS, Maron BJ. Spectrum and significance of electrocardiographic patterns, troponin levels, and thrombolysis in myocardial infarction frame count in patients with stress (tako-tsubo) cardiomyopathy and comparison to those in patients with ST-elevation anterior wall myocardial infarction. Am J Cardiol 2008; 101: 1723-8.

6. Nef HM, Möllmann H, Akashi YJ, Hamm CW. Mechanisms of stress (Takotsubo) cardiomyopathy Nat Rev Cardiol 2010; 7: 187-93.

7. Gianni M, Dentali F, Grandi AM, Sumner G, Hiralal R, Lonn E. Apical ballooning syndrome or takotsubo cardiomyopathy: a systematic review. Eur Heart J 2006; 27: 1523-29.

8. Elesber AA, Prasad A, Lennon RJ, Wright RS, Lerman A, Rihal CS. Four-year recurrence rate and prognosis of the apical ballooning syndrome. J Am Coll Cardiol 2007; 50: 448-52.

9. Tsai TT, Nallamothu BK, Prasad A, Saint S, Bates ER. Clinical problem-solving. A change of heart. N Engl J Med 2009; 361: 1010-6.

10. Eitel I, Behrendt F, Schindler K, Kivelitz D, Gutberlet $\mathrm{M}$, Schuler G, et al. Differential diagnosis of suspected apical ballooning syndrome using contrast-enhanced magnetic resonance imaging. Eur Heart J 2008; 29: 2651-9. 